\title{
KETAKSAMAAN TIPE LEMAH UNTUK OPERATOR MAKSIMAL DI RUANG MORREY TAK HOMOGEN YANG DIPERUMUM
}

\author{
Sri Maryani \\ Universitas Jenderal Soedirman \\ sri.maryani@unsoed.ac.id
}

\begin{abstract}
We discuss in this paper a weak type ( $p, p)$ inequality (where $1 \leq p<\infty)$ for maximal operator on generalized non homogeneous Morrey spaces. Our proof uses the result of Garcia-Cuerva dan Martell (2011).
\end{abstract}

Keywords: weak type inequality, maximal operator,generalized non homogeneous Morrey spaces

ABSTRAK. Pada makalah ini dibahas ketaksamaan tipe lemah ( $p$, $p)($ dengan $1 \leq p<\infty)$ untuk operator maksimal di ruang Morrey tak homogen yang diperumum. Bukti ketaksamaan menggunakan hasil dari Garcia-Cuerva dan Martell (2011).

Kata Kunci: ketaksamaan tipe lemah, operator maksimal, ruang Morrey tak homogen yang diperumum

\section{PENDAHULUAN}

Misalkan $\mu$ adalah ukuran Borel di ruang Euclid $\mathbf{R}^{d}$. Ruang $\left(\mathbf{R}^{d}, \mu\right)$ dikatakan ruang bertipe tak homogen apabila ukuran $\mu$ memenuhi growth condition orde $n$ (dengan $0<n \leq d)$ :

$$
\mu(B(x, r)) \leq C r^{n}
$$

untuk setiap bola buka $B(x, r)$ yang berpusat di $x$ dan berjari-jari $r$ (Nazarov, dkk., 1998). Catat bahwa konstanta positif $C$ pada growth condition (1) tidak bergantung pada $x$ maupun $r$. Beberapa hasil penelitian di ruang bertipe tak homogen dapat 
dilihat pada (Gunawan, dkk., 2009; Sawano, 2005; Sihwaningrum, 2010; Terasawa, 2006).

Di ruang bertipe tak homogen, didefinisikan operator $M_{p}^{n}$ (untuk $1 \leq p<\infty$ dan $0<n \leq d$ ) dengan

$$
M_{p}^{n} f(x):=\left(M|f|^{p}(x)\right)^{1 / p}=\sup _{r>0}\left(\frac{1}{r^{n}} \int_{B(x, r)}|f(y)|^{p} d \mu(y)\right)^{1 / p} .
$$

Definisi ini analog dengan definisi serupa pada (Nakai, 1994) di ruang bertipe homogen. Jika $p=1$, operator $M_{p}^{n}$ tidak lain adalah operator $M^{n}$, yang diperkenalkan oleh Garcia-Cuerva dan Martell (2001). Operator $M^{n}$ memenuhi ketaksamaan tipe lemah $(1,1)$ di ruang Lebesgue tak homogen. Selain itu, $M^{n}$ juga memenuhi ketaksamaan terboboti berikut ini.

Teorema 1.1. (Garcia-Cuerva dan Martell, 2001) Untuk bobot $w$ dan sembarang fungsi fdi $\mathbf{R}^{d}$ berlaku

$$
\int_{\left\{x \in \mathbf{R}^{d}: M^{n} f(x)>\gamma\right\}} w(x) d \mu(x) \leq \frac{C}{\gamma} \int_{\mathbf{R}^{d}}|f(x)| M^{n} w(x) d \mu(x)
$$

dengan $C$ adalah konstanta positif yang tidak bergantung pada f maupun $w$.

Dengan menggunakan Teorema 1.1, pada makalah ini akan dibuktikan ketaksamaan tipe lemah $(p, p)$ (dengan $1 \leq p<\infty)$ untuk operator maksimal $M_{p}^{n}$ di ruang Morrey tak homogen yang diperumum.

\section{HASIL DAN PEMBAHASAN}

Misalkan $\phi:(0, \infty) \rightarrow(0, \infty)$ adalah fungsi yang hampir turun, yaitu terdapat konstanta $C>0$ sehingga $\phi(t) \leq C \phi(r)$ apabila $t>s$. Untuk $1 \leq p<\infty$, ruang 
Morrey tak homogen yang diperumum $\mathfrak{M}^{p, \phi}(\mu)=\mathfrak{W}^{p, \phi}\left(\mathbf{R}^{d}, \mu\right)$ didefinisikan sebagai ruang dari semua fungsi $f \in L_{\text {loc }}^{p}(\mu)$ dengan

$$
\left\|f: \mathfrak{W}^{p, \phi}(\mu)\right\|:=\sup _{r>0} \frac{1}{\phi(r)}\left(\frac{1}{r^{n}} \int_{B(x, r)}|f(y)|^{p}\right)^{1 / p}<\infty .
$$

Apabila $\phi(t)=t^{-n / p}$, maka ruang Morrey tak homogen merupakan ruang Lebesgue tak homogen, yakni $\mathfrak{W}^{p, \phi}(\mu)=L^{p}(\mu)$. Sementara itu, sifat-sifat lain dari ruang Morrey tak homogen yang diperumum dapat dilihat pada (Sihwaningrum, dkk., 2008a dan 2008b).

Kemudian, dengan menggunakan Teorema 1.1, diperoleh ketaksamaan tipe lemah $(p, p)$ untuk operator maksimal.

Teorema 2.1. Untuk $\phi:(0, \infty) \rightarrow(0, \infty)$ diasumsikan bahwa $\phi$ merupakan fungsi yang hampir turun. Jika terdapat konstanta $C_{1}>0$ sehingga untuk setiap $r>0$ fungsi $\phi$ memenuhi

$$
\int_{r}^{\infty} \frac{[\phi(t)]^{p}}{t} d t \leq C_{1}[\phi(r)]^{p}
$$

dengan $1 \leq p<\infty$, maka terdapat konstanta $C>0$ sehingga untuk setiap $\gamma>0$ dan untuk setiap bola $B(a, r)$ berlaku ketaksamaan

$$
\mu\left\{x \in B(a, r): M_{p}^{n} f(x)>\gamma\right\} \leq \frac{C}{\gamma^{p}} r^{n}(\phi(r))^{p}\left\|f: \mathfrak{M}^{p, \phi}(\mu)\right\|^{p}
$$

Bukti. Misalkan $\chi_{B(a, r)}$ merupakan fungsi karakteristik dari $B(a, r)$. Untuk setiap $f \in \mathfrak{M}^{p, \phi}$, berlaku

$$
\int_{\mathbb{R}^{d}}|f(x)|^{p} d x M \chi_{B(a, r)}
$$


$\leq \int_{B(a, 2 r)}|f(x)|^{p} d x M \chi_{B(a, r)} d \mu+\sum_{k=1}^{\infty} \int_{B\left(a, 2^{k+1} r\right) \backslash B\left(a, 2^{k} r\right)}|f(x)|^{p} d x M \chi_{B(a, r)} d \mu$

$\leq C\left[\int_{B(a, 2 r)}|f(x)|^{p} d x M \chi_{B(a, r)} d \mu+\sum_{k=1}^{\infty} \int_{B\left(a, 2^{k+1} r\right) \backslash B\left(a, 2^{k} r\right)}|f(x)|^{p} 2^{-k n} d \mu\right]$

$\leq C\left\{(2 r)^{n}(\phi(2 r))^{p}\left\|f: \mathfrak{M}^{p, \phi}\right\|^{p}\right.$

$$
\left.+\sum_{k=1}^{\infty} 2^{-k n}\left(2^{k+1} r\right)^{n}\left(\phi\left(2^{k+1} r\right)\right)^{p}\left\|f: \mathfrak{M}^{p, \phi}\right\|^{p}\right\}
$$

$=C r^{n}\left\|f: \mathfrak{M}^{p, \phi}\right\|^{p}\left\{(\phi(2 r))^{p}+\sum_{k=1}^{\infty}\left(\phi\left(2^{k+1} r\right)\right)^{p}\right\}$

$=\operatorname{Cr}^{n}\left\|f: \mathfrak{M}^{p, \phi}\right\|^{p} \sum_{k=0}^{\infty}\left(\phi\left(2^{k+1} r\right)\right)^{p}$

$\leq C r^{n}\left\|f: \mathfrak{M}^{p, \phi}\right\|^{p} \sum_{k=0}^{\infty}\left(\phi\left(2^{k} r\right)\right)^{p}$.

Karena $\phi$ fungsi yang hampir turun, maka

$$
\begin{aligned}
\int_{\mathbb{R}^{d}}|f(x)|^{p} d x M \chi_{B(a, r)} d \mu & \leq C r^{n}\left\|f: \mathfrak{M}^{p, \phi}\right\|^{p} \sum_{k=0}^{\infty} \int_{2^{k}}^{2^{k+1}} \frac{(\phi(t))^{p}}{t} d t \\
& \leq C r^{n}\left\|f: \mathfrak{M}^{p, \phi}\right\|^{p} \sum_{k=0}^{\infty} \int_{r}^{\infty} \frac{(\phi(t))^{p}}{t} d t \\
& \leq C r^{n}\left\|f: \mathfrak{M}^{p, \phi}\right\|^{p}(\phi(r))^{p} .
\end{aligned}
$$

Selanjutnya, dengan Teorema 1.1, terbukti bahwa

$$
\mu\left\{x \in B: M_{p}^{n} f(x)>\gamma\right\}=\int_{\left\{x: M|f|^{p}(x)>\gamma^{p}\right\}} \chi_{B(a, r)}(x) d \mu
$$




$$
\begin{aligned}
& \leq \frac{C}{\gamma^{p}} \int_{\mathbb{R}^{d}}|f(x)|^{p} M \chi_{B(a, r)}(x) d \mu \\
& \leq \frac{C r^{n}(\phi(r))^{p}}{\gamma^{p}}\left\|f: \mathfrak{M}^{p, \phi}(\mu)\right\|^{p} \cdot \mathbf{\square}
\end{aligned}
$$

\section{KESIMPULAN DAN SARAN}

Hasil yang diperoleh pada Teorema 2.1 analog dengan hasil dari Nakai (1994) di ruang Morrey homogeny yang diperumum. Selain itu, teorema tersebut merupakan perumuman dari Teorema 2.1 pada (Sihwaningrum, dkk., 2012).

\section{UCAPAN TERIMAKASIH}

Penelitian ini dibiayai oleh DIPA Universitas Jenderal Soedirman melalui Hibah Fundamental dengan kontrak No. 1055.04/UN23.9/PN/2012

\section{DAFTAR PUSTAKA}

Garcia-Cuerva, J. dan Martell, J.M. (2001) Two-Weight Norm Inequalities for Maximal Operators and Fractional Integral on Non-Homogeneous Spaces, Indiana Univ. Math.s J. 50(3), 1241-1280.

Gunawan, H., Sawano, Y. dan Sihwaningrum, I. (2009) Fractional Integral Operators in Non-Homogeneous Spaces, Bull. Aust. Math. Soc. 80(2), 324334.

Nakai, E. (1994) Hardy-Littlewood maximal operator, singular integral operators, and Riesz potential on generalized Morrey spaces, Math. Nachr. 166, 95-103.

Nazarov, F., Treil, S. dan Volberg, A. (1998) Weak Type Estimates and Cotlar Inequalities for Calderón-Zygmund Operators on Nonhomogeneous Space, Internat. Math. Res. Notices 9, 463-487.

Sawano, Y. (2005) Sharp Estimates of the Modified Hardy Littlewood Maximal Operator on the Non-homogeneous Space via Covering Lemmas, Hokkaido Math. J. 34, 435-458. 
Sihwaningrum, I., Gunawan, H. dan Budhi, W.S. (2008a) Operator Integral Fraksional dan Ketaksamaan Olsen di Ruang Morrey Tak Homogen yang Diperumum, Prosiding Seminar Nasional Mahasiswa S3 Matematika seIndonesia, Universitas Gadjah mada, Yogyakarta, 39-47.

Sihwaningrum, I., Gunawan, H., Soeharyadi, Y. dan W.S. Budhi. (2008b) Generalized Non-homogeneous Morrey Spaces and Olsen Inequality, Prosiding Seminar Nasional Matematika dan Pendidikan Matematika, Universitas Negeri Yogyakarta, Yogyakarta, 1-7.

Sihwaningrum, I., Maryani, S, dan Gunawan, H. (2012) Weak Type Inequalities for Fractional Integral Operators on Generalized Non-homogeneous Morrey Spaces, Analysis in Theory and Applications, 28, 65-72

Terasawa, Y. (2006) Outer Measures and Weak Type $(1,1)$ Estimates of HardyLittlewood Maximal Operators, Journal of Inequalities and Applications. 46, $471-497$. 